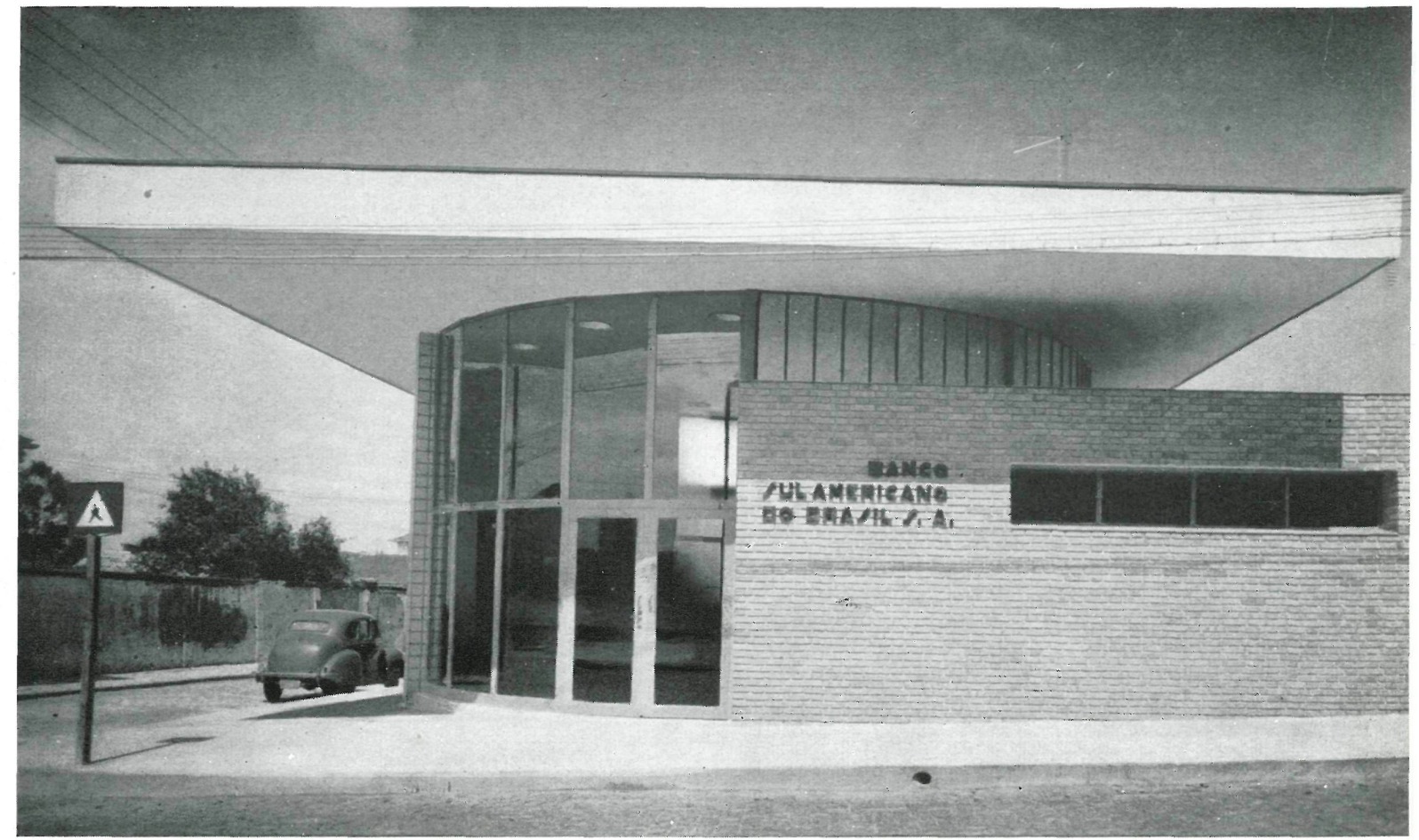

\title{
Agencia de la Banca Sud-americana del Brasil, s. en Capivari
}

RINO LEVI, ROBERTO CERQUEIRA CESAR Y LUIS ROBERTO CARVALHO FRANCO, arquitectos

\section{sinopsis}

El edificio consta de dos cuerpos: uno para las instalaciones de la Banca, y el otro para la vivienda del gerente. La estructura es mixta, a base de hormigón armado y acero; los muros, de ladrillo; el techo, ejecutado en losa de hormigón armado y ladrillos huecos, y la cubierta es de fibrocemento ondulado. Los muros de los cuerpos de la Banca han sido revestidos, interior y exteriormente, con baldosas de cemento, de $15 \times 15 \mathrm{~cm}$, del tipo emplead para el suelo. El dibujo es gris sobre fondo blanco. El techo de la sala de la Banca va acondicionado con material acustico (a base de $2,5 \mathrm{~cm}$ de lana de vidrio sobre placas perforadas de Duralex) para reducir la reverberación. Esta medida venía impuesta dada la altura relativamente grande $y$ los muros en curva revestidos de material lise y duro.

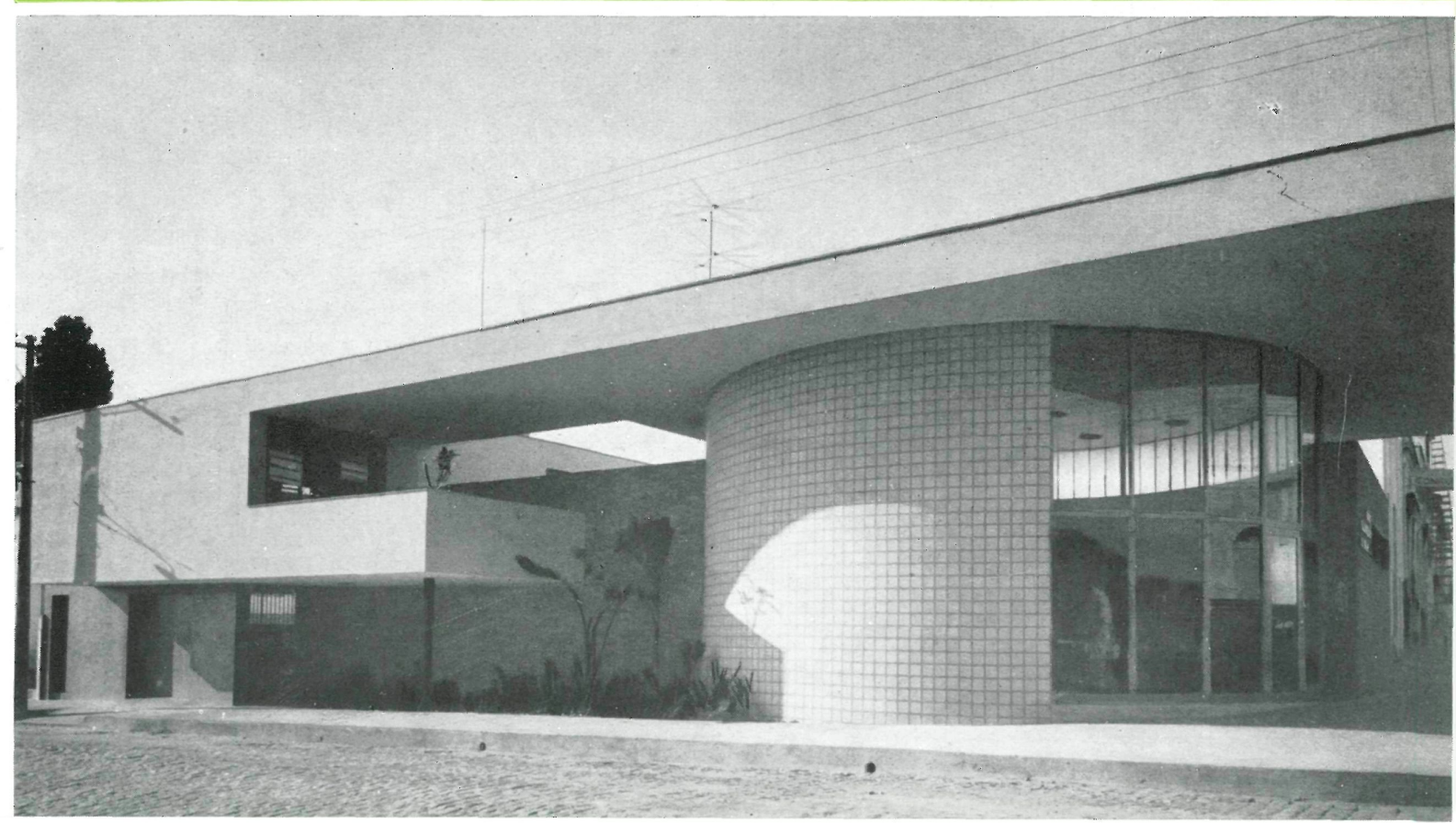


El edificio consta de dos cuerpos: uno para las instalaciones de la Banca, y el otro para vivienda del director.

Este último está organizado en dos plantas: la inferior, ocupada por el garaje, locales de servicio y jardín. Y la superior, por el comedor-sala de estar, con amplia terraza, tres dormitorios, baño y cocina.

En la zona de la Banca destaca la atractiva disposición en planta circular del área de despacho al público, que se proyecta en el alzado como un volumen cilindrico.

Dicha área está en conexión, por un lado, con un patio interior ajardinado $\mathrm{y}$, por el otro, con el despacho del director, cámara acorazada, servicios y archivos. Los paneles divisorios han sido realizados a base de bastidores metálicos y vidrios translúcidos.

La organización del conjunto en torno a un patio interior constituye un acierto, pues las zonas ajardinadas, el parterre existente y la creación de espacios sombreados producen unas diferencias de temperatura que ayudan a la ventilación natural de las distintas dependencias, creando así un óptimo ambiente de trabajo y rendimiento.

La estructura es mixta, a base de hormigón armado y acero; los muros son de ladrillo; el techo está formado por una placa aligerada de hormigón armado, y la cubierta, a base de fibrocemento ondulado.

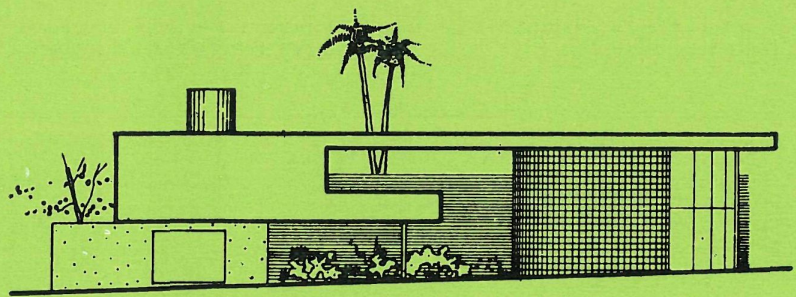

alzendo

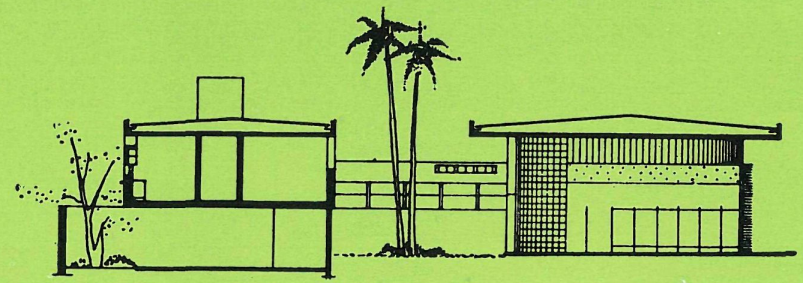

SECEIĆm
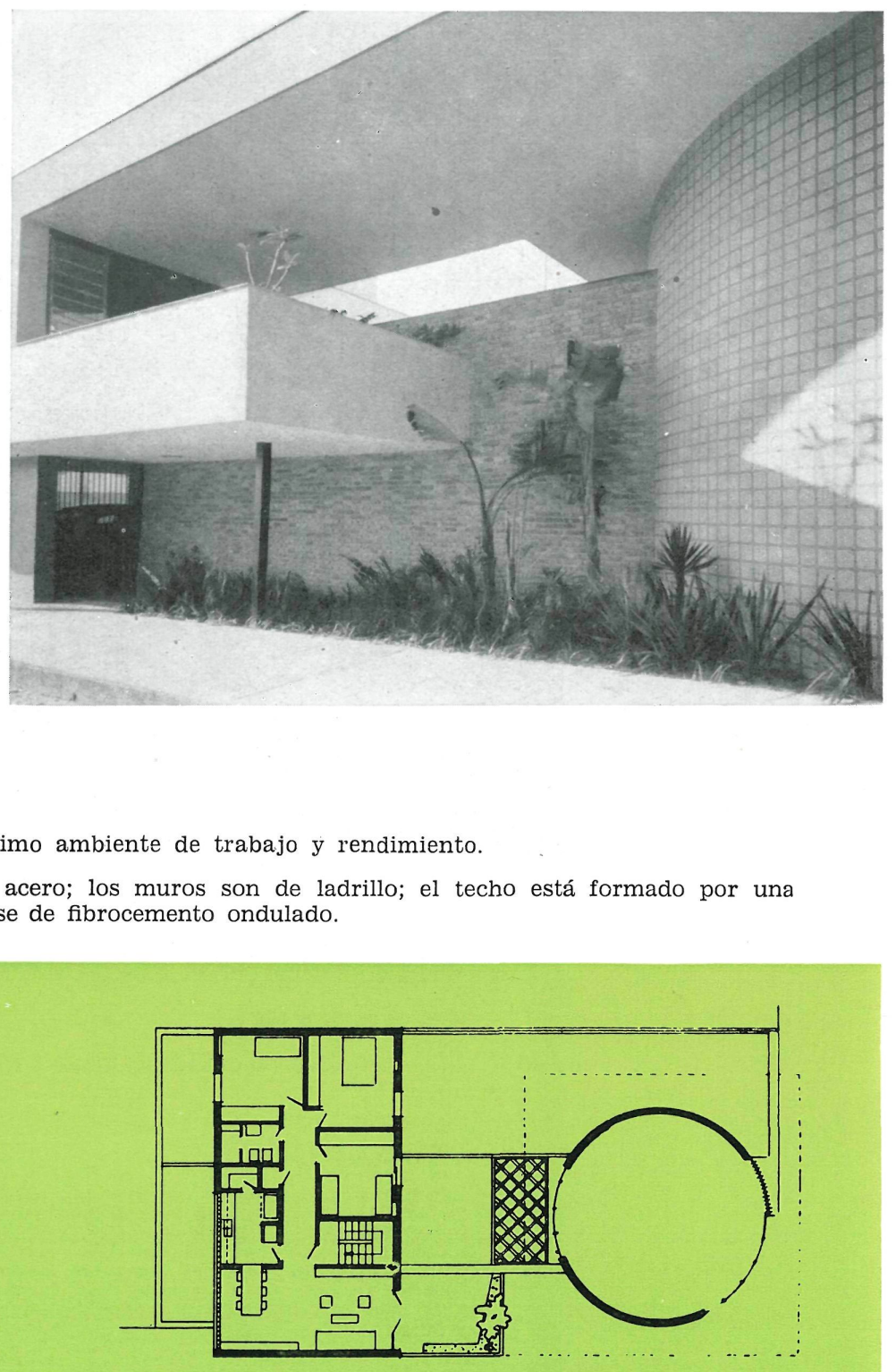

plemen proimernen

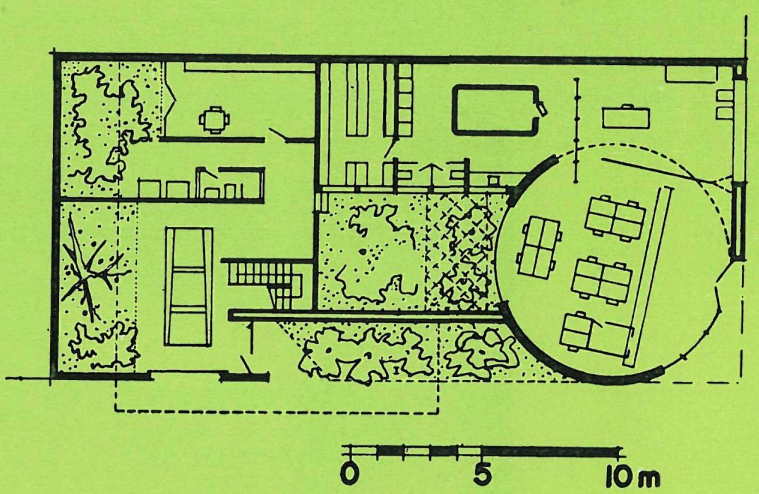



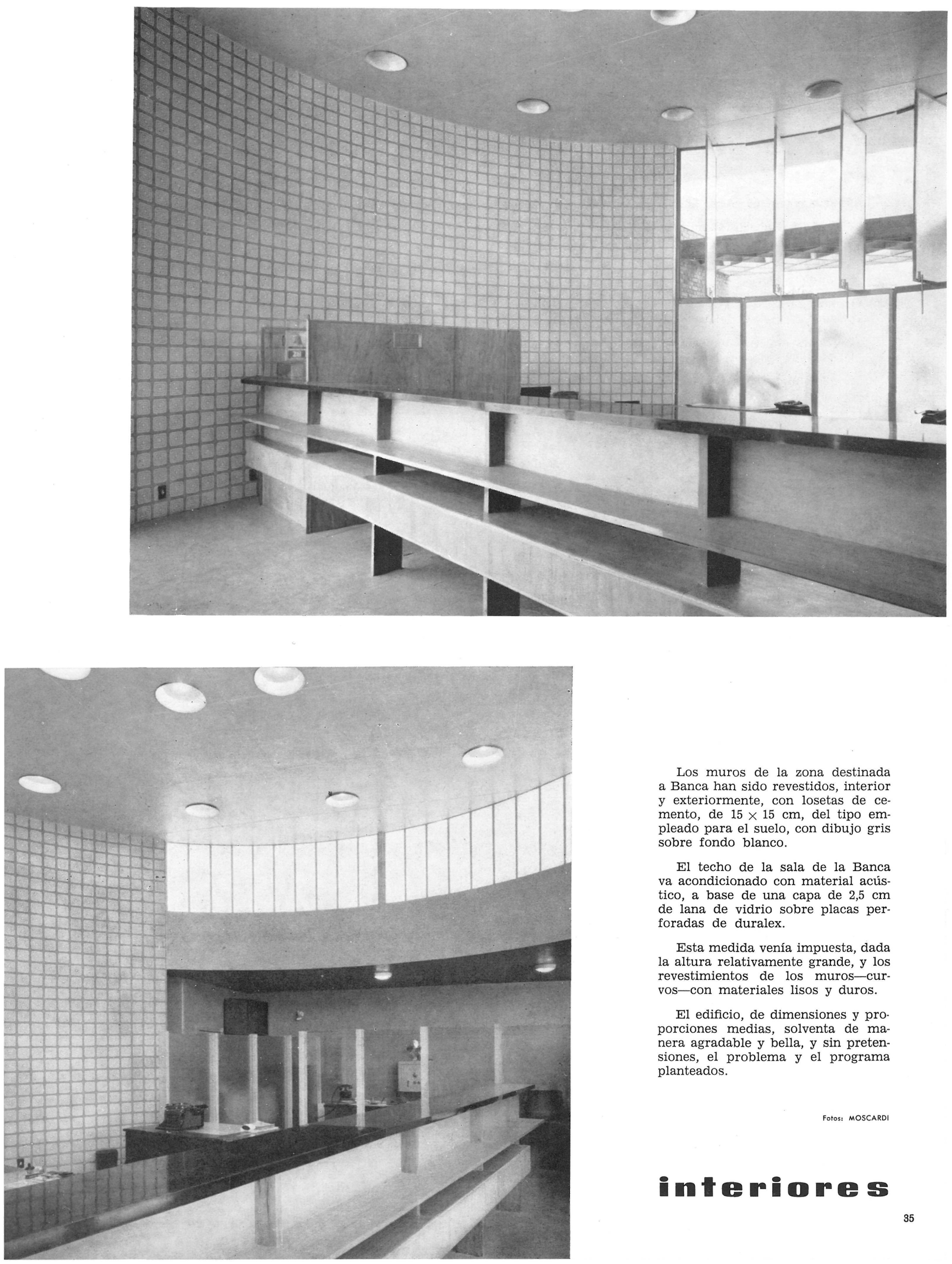

Los muros de la zona destinada a Banca han sido revestidos, interior y exteriormente, con losetas de cemento, de $15 \times 15 \mathrm{~cm}$, del tipo empleado para el suelo, con dibujo gris sobre fondo blanco.

El techo de la sala de la Banca va acondicionado con material acústico, a base de una capa de $2,5 \mathrm{~cm}$ de lana de vidrio sobre placas perforadas de duralex.

Esta medida venía impuesta, dada la altura relativamente grande, y los revestimientos de los muros-curvos-con materiales lisos y duros.

El edificio, de dimensiones y pro. porciones medias, solventa de manera agradable y bella, y sin pretensiones, el problema y el programa planteados.

Fotos: MOSCARDI

\section{interiores}




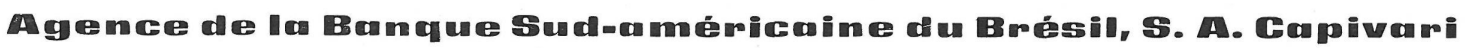
Rino Levi, Roberto Cerqueira Cesar et Luis Roberto Carvalho Franco, architectes.

L'édifice se compose de deux corps: un pour les installations de la banque et l'autre pour le logement de l'administrateur.

La structure est mixte, béton armé et acier; les murs, en brique; le plafond exécuté en dalle de béton armé et briques creuses et la couverture est en fibrociment ondulé.

Les murs de la banque ont été revêtus, tant à l'extérieur qu'à l'intérieur, de dalles de ciment, de $15 \mathrm{~cm}$ sur 15, du type employé pour le sol. Le dessin est gris sur fond blanc.

Le plafond de la salle de la banque a été conditionné à l'aide de matériaux acoustiques (à base de $2,5 \mathrm{~cm}$ de laine de verre sur des plaques percées de Duralex) pour réduire la réverbération. Cette mesure était im. posée à cause de la hauteur relativement grande et des murs courbes revêtus de matériaux lisses et durs.

\section{Branclh of the Brasil south American Bank, S. A., cat Gapivami}

Rino Levi, Roberto Cerqueira César and Luis Roberto Carvalho Franco, architects.

This building has two blocks, one for the Banks' installations and the other is the manager's house.

The building has a concrete and steel compound structure. The walls are brick, the flooring is a reinforced concrete slab and hollow bricks, and the roof consists of corrugated fibrocement sheeting.

The bank block walls have been faced, both internally and externally, with cement tiles, $15 \times 15$ cms in size, of the same type that are employed for the flooring. The design is grey on white background.

The ceiling of the bank hall is fitted with acoustical material $(2.5 \mathrm{cms}$ of glass wool on Duralex perforated slabs), to reduce reverberation. This measure was necessary because of the relatively large height of the hall. The walls are cylindrically curved and have a smooth and hard surface.

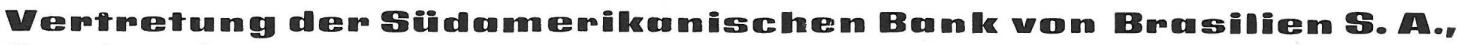 Expiven pi}

Rino Levi, Roberto Cerqueira César und Luis Roberto Calvalho Franco, Architekten.

Das Gebäude besteht aus zwei Teilen, einem für die Einrichtungen der Bank und einem anderen für die Wohnung des Geschäftsführers.

Die Struktur ist gemischt, und zwar auf Grund von Stahlbeton und Stahl, die Mauern sind aus Ziegeln, die Decke in Stahlbetonfliesen und Hohlziegeln ausgeführt, und das Dach besteht aus Wellasbestzement.

Die Wände des Bankgebäudes wurden innen und aussen mit Zement-Fliesen von $15 \times 15$ cm verkleidet, gleicher Art wie die für den Fussboden verwendeten. Ihr Muster ist ein graues auf weissem Grunde.

Die Decke des Bankraumes ist mit akustischem Material versehen (auf Grund von 2,5 cm Glaswolle auf durchlöcherten Platten aus Duralex), um den Schall zu vermindern. Diese Massnahme wurde wegen der verhältnismässig grossen Höhe und der gebogenen, mit glattem und hartem Material verkleideten Wände getroffen. 